\title{
Soft Ideal Theory Soft Local Function and Generated Soft Topological Spaces
}

\author{
A. Kandil ${ }^{1, *}$, O. A. E. Tantawy ${ }^{2}$, S. A. El-Sheikh ${ }^{3}$ and A. M. Abd El-latif ${ }^{3}$ \\ ${ }^{1}$ Mathematics Department, Faculty of Science, Helwan University, Helwan, Egypt \\ ${ }^{2}$ Mathematics Department, Faculty of Science, Zagazig University, Zagazig, Egypt \\ ${ }^{3}$ Mathematics Department, Faculty of Education, Ain Shams University, Cairo, Egypt
}

Received: 14 Jul. 2013, Revised: 16 Oct. 2013, Accepted: 17 Oct. 2013

Published online: 1 Jul. 2014

\begin{abstract}
The purpose of this paper is to introduce the notion of soft ideal in soft set theory. The concept of soft local function is also introduced. These concepts are discussed with a view to find new soft topologies from the original one. The basic structure, especially a basis for such generated soft topologies also studied here. Finally, the notion of compatibility of soft ideals with soft topologies is introduced and some equivalent conditions concerning this topic are established here.
\end{abstract}

Keywords: Soft set, Soft topological space, Soft interior, Soft closure, Open soft, Closed soft, Soft ideal, Soft local function, *-soft topology, Compatible soft ideal.

\section{Introduction}

The concept of soft sets was first introduced by Molodtsov [19] in 1999 as a general mathematical tool for dealing with uncertain objects. In [19,18], Molodtsov successfully applied the soft theory in several directions, such as smoothness of functions, game theory, operations research, Riemann integration, Perron integration, probability, theory of measurement, and so on. After presentation of the operations of soft sets [16], the properties and applications of soft set theory have been studied increasingly $[3,13,18,22]$. In recent years, many interesting applications of soft set theory have been expanded by embedding the ideas of fuzzy sets $[1,2,6,14$, $15,16,17,18,20,27]$. To develop soft set theory, the operations of the soft sets are redefined and a uni-int decision making method was constructed by using these new operations [7].

Recently, in 2011, Shabir and Naz [23] initiated the study of soft topological spaces. They defined soft topology $\tau$ on the collection of soft sets over $X$. Consequently, they defined basic notions of soft topological spaces such as open soft and closed sets, soft subspace, soft closure, soft nbd of a point, soft separation axioms, soft regular spaces and soft normal spaces and established many of their properties. Hussain and Ahmad [9] investigated the properties of open (closed) soft, soft nbd and soft closure. They also defined and discussed the properties of soft interior, soft exterior and soft boundary which are fundamental for further research on soft topology and will strengthen the foundations of the theory of soft topological spaces. Kandil et al. [11] introduced a unification of some types of different kinds of subsets of soft topological spaces using the notion of $\gamma$-operation. The purpose of this paper is to introduce the notion of soft ideal in soft set theory. The concept of soft local function is also introduced. These concepts are discussed with a view to find new soft topologies from the original one. The basic structure, especially a basis for such generated soft topologies also studied here. Finally, the notion of

*Corresponding author e-mail: Alaa_8560@yahoo.com,dr.Alaa_daby@yahoo.com 
compatibility of soft ideals with soft topologies is introduced and some equivalent conditions concerning this topic are established here.

\section{Preliminaries}

Definition 2.1. [19] Let $X$ be an initial universe and $E$ be a set of parameters. Let $P(X)$ denote the power set of $X$ and $A$ be a non-empty subset of $E$. A pair $(F, A)$ denoted by $F_{A}$ is called a soft set over $X$, where $F$ is a mapping given by $F: A \rightarrow P(X)$. In other words, a soft set over $X$ is a parametrized family of subsets of the universe $X$. For a particular $e \in A, F(e)$ may be considered the set of $e$ approximate elements of the soft set $(F, A)$ and if $e \notin A$, then $F(e)=\phi$ i.e

$F_{A}=\{F(e): e \in A \subseteq E, F: A \rightarrow P(X)\}$. The family of all these soft sets denoted by $S S(X)_{A}$.

Definition 2.2.[16] Let $F_{A}, G_{B} \in S S(X)_{E}$. Then $F_{A}$ is soft subset of $G_{B}$, denoted by $F_{A} \subseteq G_{B}$, if

(1) $A \subseteq B$, and

(2) $F(e) \subseteq G(e), \forall e \in A$.

In this case, $F_{A}$ is said to be a soft subset of $G_{B}$ and $G_{B}$ is said to be a soft superset of $F_{A}, G_{B} \tilde{\supseteq} F_{A}$.

Definition 2.3.[16] Two soft subset $F_{A}$ and $G_{B}$ over a common universe set $X$ are said to be soft equal if $F_{A}$ is a soft subset of $G_{B}$ and $G_{B}$ is a soft subset of $F_{A}$.

Definition 2.4.[3] The complement of a soft set $(F, A)$, denoted by $(F, A)^{\prime}$, is defined by $(F, A)^{\prime}=\left(F^{\prime}, A\right)$, $F^{\prime}: A \rightarrow P(X)$ is a mapping given by $F^{\prime}(e)=X-F(e)$, $\forall e \in A$ and $F^{\prime}$ is called the soft complement function of $F$.

Clearly $\left(F^{\prime}\right)^{\prime}$ is the same as $F$ and $\left((F, A)^{\prime}\right)^{\prime}=(F, A)$.

Definition 2.5.[23] The difference of two soft sets $(F, E)$ and $(G, E)$ over the common universe $X$, denoted by $(F, E)-(G, E)$ is the soft set $(H, E)$ where for all $e \in E$, $H(e)=F(e)-G(e)$.

Definition 2.6.[23] Let $(F, E)$ be a soft set over $X$ and $x \in$ $X$. We say that $x \in(F, E)$ read as $x$ belongs to the soft set $(F, E)$ whenever $x \in F(e)$ for all $e \in E$.

Definition 2.7.[16] A soft set $(F, A)$ over $X$ is said to be a NULL soft set denoted by $\tilde{\phi}$ or $\phi_{A}$ if for all $e \in A, F(e)=\phi$ (null set).

Definition 2.8.[16] A soft set $(F, A)$ over $X$ is said to be an absolute soft set denoted by $\tilde{A}$ or $X_{A}$ if for all $e \in A, F(e)=$ $X$. Clearly we have $X_{A}^{\prime}=\phi_{A}$ and $\phi_{A}^{\prime}=X_{A}$.

Definition 2.9.[16] The union of two soft sets $(F, A)$ and $(G, B)$ over the common universe $X$ is the soft set $(H, C)$, where $C=A \cup B$ and for all $e \in C$,

$$
H(e)=\left\{\begin{array}{l}
F(e), e \in A-B, \\
G(e), e \in B-A, \\
F(e) \cup G(e), e \in A \cap B .
\end{array}\right.
$$

Definition 2.10.[16] The intersection of two soft sets $(F, A)$ and $(G, B)$ over the common universe $X$ is the soft set $(H, C)$, where $C=A \cap B$ and for all $e \in C$, $H(e)=F(e) \cap G(e)$. Note that, in order to efficiently discuss, we consider only soft sets $(F, E)$ over a universe $X$ in which all the parameter set $E$ are same. We denote the family of these soft sets by $S S(X)_{E}$.

Definition 2.11.[28] Let $I$ be an arbitrary indexed set and $L=\left\{\left(F_{i}, E\right), i \in I\right\}$ be a subfamily of $S S(X)_{E}$.

(1)The union of $L$ is the soft set $(H, E)$, where $H(e)=\bigcup_{i \in I} F_{i}(e)$ for each $e \in E$. We write $\tilde{U}_{i \in I}\left(F_{i}, E\right)=(H, E)$.

(2)The intersection of $L$ is the soft set $(M, E)$, where $M(e)=\bigcap_{i \in I} F_{i}(e)$ for each $e \in E$. We write $\tilde{\bigcap}_{i \in I}\left(F_{i}, E\right)=(M, E)$.

Definition 2.12.[23] Let $\tau$ be a collection of soft sets over a universe $X$ with a fixed set of parameters $E$, then $\tau \subseteq$ $S S(X)_{E}$ is called a soft topology on $X$ if

(1) $\tilde{X}, \tilde{\phi} \in \tau$, where $\tilde{\phi}(e)=\phi$ and $\tilde{X}(e)=X, \forall e \in E$,

(2)the union of any number of soft sets in $\tau$ belongs to $\tau$,

(3)the intersection of any two soft sets in $\tau$ belongs to $\tau$.

The triplet $(X, \tau, E)$ is called a soft topological space over $X$.

Definition 2.13.[9] Let $(X, \tau, E)$ be a soft topological space. A soft set $(F, A)$ over $X$ is said to be closed soft set in $X$, if its relative complement $(F, A)^{\prime}$ is an open soft set.

Definition 2.14.[9] $\operatorname{Let}(X, \tau, E)$ be a soft topological space. The members of $\tau$ are said to be open soft sets in $X$. We denote the set of all open soft sets over $X$ by $O S(X, \tau, E)$, or $O S(X)$ and the set of all closed soft sets by $C S(X, \tau, E)$, or $C S(X)$.

Definition 2.15.[23] Let $(X, \tau, E)$ be a soft topological space and $(F, E) \in S S(X)_{E}$. The soft closure of $(F, E)$, denoted by $\operatorname{cl}(F, E)$ is the intersection of all closed soft super sets of $(F, E)$ i.e
$\operatorname{cl}(F, E)$
$=$
$\tilde{\cap}\{(H, E)$

$(H, E)$ is closed soft set and $(F, E) \tilde{\simeq}(H, E)\})$.

Definition 2.16.[28] Let $(X, \tau, E)$ be a soft topological space and $(F, E) \in S S(X)_{E}$. The soft interior of $(G, E)$, denoted by $\operatorname{int}(G, E)$ is the union of all open soft subsets of $(G, E)$ i.e $\operatorname{int}(G, E)=\tilde{\cup}\{(H, E)$ : $(H, E)$ is an open soft set and $(H, E) \tilde{\subseteq}(G, E)\})$. 
Definition 2.17.[28] The soft set $(F, E) \in S S(X)_{E}$ is called a soft point in $X_{E}$ if there exist $x \in X$ and $e \in E$ such that $F(e)=\{x\}$ and $F\left(e^{\prime}\right)=\phi$ for each $e^{\prime} \in E-\{e\}$, and the soft point $(F, E)$ is denoted by $x_{e}$.

Proposition 2.1.[24] The union of any collection of soft points can be considered as a soft set and every soft set can be expressed as union of all soft points belonging to it.

Definition 2.18.[28] The soft point $x_{e}$ is said to be belonging to the soft set $(G, A)$, denoted by $x_{e} \tilde{\in}(G, A)$, if for the element $e \in A, F(e) \subseteq G(e)$.

Definition 2.19.[28] A soft set $(G, E)$ in a soft topological space $(X, \tau, E)$ is called a soft neighborhood (briefly: nbd) of the soft point $x_{e} \tilde{\in} X_{E}$ if there exists an open soft set $(H, E)$ such that $x_{e} \tilde{\in}(H, E) \tilde{\subseteq}(G, E)$.

A soft set $(G, E)$ in a soft topological space $(X, \tau, E)$ is called a soft neighborhood of the soft $(F, E)$ if there exists an open soft set $(H, E)$ such that $(F, E) \tilde{\epsilon}(H, E) \tilde{\subseteq}(G, E)$. The neighborhood system of a soft point $x_{e}$, denoted by $N_{\tau}\left(x_{e}\right)$, is the family of all its neighborhoods.

Theorem 2.1.[25] Let $(X, \tau, E)$ be a soft topological space. A soft point $e_{x} \tilde{\epsilon} c l(F, E)$ if and only if each soft neighborhood of $e_{x}$ intersects $(F, E)$.

Definition 2.20.[21] Let $(X, \tau, E)$ be a soft topological space and $(F, E) \in S S(X)_{E}$. Define $\tau_{(F, E)}=\{(G, E) \tilde{\cap}(F, E):(G, E) \in \tau\}$, which is a soft topology on $(F, E)$. This soft topology is called soft relative topology of $\tau$ on $(F, E)$, and $\left[(F, E), \tau_{(F, E)}\right]$ is called soft subspace of $(X, \tau, E)$.

Definition 2.21.[28] Let $S S(X)_{A}$ and $S S(Y)_{B}$ be families of soft sets, $u: X \rightarrow Y$ and $p: A \rightarrow B$ be mappings. Then the mapping $f_{p u}: S S(X)_{A} \rightarrow S S(Y)_{B}$ is defined as:

(1)If $(F, A) \in S S(X)_{A}$. Then the image of $(F, A)$ under $f_{p u}$, written as $f_{p u}(F, A)=\left(f_{p u}(F), p(A)\right)$, is a soft set in $\operatorname{SS}(Y)_{B}$ such that

$$
\begin{aligned}
& f_{p u}(F)(b) \\
& \begin{cases}\cup_{x \in p^{-1}(b) \cap A} & u(F(a)), \quad p^{-1}(b) \cap A \neq \phi, \\
\phi, & \text { otherwise. }\end{cases} \\
& \text { for all } b \in B .
\end{aligned}
$$

(2)If $(G, B) \in S S(Y)_{B}$. Then the inverse image of $(G, B)$ under $f_{p u}$, written as $f_{p u}^{-1}(G, B)=\left(f_{p u}^{-1}(G), p^{-1}(B)\right)$, is a soft set in $\operatorname{SS}(X)_{A}$ such that

$$
f_{p u}^{-1}(G)(a)= \begin{cases}u^{-1}(G(p(a))), & p(a) \in B, \\ \phi, & \text { otherwise. }\end{cases}
$$

for all $a \in A$.

Definition 2.22.[28] Let $(X, \tau, A)$ and $\left(Y, \tau^{*}, B\right)$ be soft topological spaces and $f_{p u}: S S(X)_{A} \rightarrow S S(Y)_{B}$ be a function. Then
(1)The function $f_{p u}$ is called continuous soft (cts-soft) if $f_{p u}^{-1}(G, B) \in \tau \forall(G, B) \in \tau^{*}$.

(2)The function $f_{p u}$ is called open soft if $f_{p u}(G, A) \in \tau^{*} \forall(G, A) \in \tau$.

Definition 2.23.[5] Let $(X, \tau, E)$ be a soft topological space and $x, y \in X$ such that $x \neq y$. Then $(X, \tau, E)$ is called soft Hausdorff space or soft $T_{2}$ space if there exist open soft sets $(F, E)$ and $(G, E)$ such that $x \in(F, E), y \in(G, E)$ and $(F, E) \tilde{\cap}(G, E)=\tilde{\phi}$

Definition 2.24.[10]. A non-empty collection $I$ of subsets of a set $X$ is called an ideal on $X$, if it satisfies the following conditions

(1) $A \in I$ and $B \in I \Rightarrow A \cup B \in I$,

(2) $A \in I$ and $B \subseteq A \Rightarrow B \in I$,

i.e. $I$ is closed under finite unions and subsets.

\section{Soft ideal, soft local function and generated soft topology}

In this section We generate a soft topological space finer than the given soft topological space $(X, \tau, E)$ on the same set $X$ with a fixed set of parameters $E$ by using the soft ideal notion. We denote $(X, \tau, E, \tilde{I})$ as a soft topological space with soft ideal $\tilde{I}$ on $X$.

Definition 3.1. Let $\tilde{I}$ be a non-null collection of soft sets over a universe $X$ with the same set of parameters $E$. Then $\tilde{I} \subseteq S S(X)_{E}$ is called a soft ideal on $X$ with the same set $E$ if

$(1)(F, E) \in \tilde{I}$ and $(G, E) \in \tilde{I} \Rightarrow(F, E) \widetilde{\cup}(G, E) \in \tilde{I}$,

(2) $(F, E) \in \tilde{I}$ and $(G, E) \tilde{\subseteq}(F, E) \Rightarrow(G, E) \in \tilde{I}$,

i.e. $\tilde{I}$ is closed under finite soft unions and soft subsets.

Example 3.1. Let $X$ be a universe set. Then each of the following families is a soft ideal over $X$ with the same set of parameters $E$,

(1) $\tilde{I}=\{\tilde{\phi}\}$,

$(2) \tilde{I}=\operatorname{SS}(X)_{E}=\{(F, E) \quad:$ $(F, E)$ is a soft set over $X$ with the fixed set of parameters $E\}$,

(3) $\tilde{I}_{f}=\left\{(F, E) \in S S(X)_{E}:(F, E)\right.$ is a finite soft set $\}$, called soft ideal of finite soft subsets of $\tilde{X}$,

(4) $\tilde{I}_{C}=\left\{(F, E) \in S S(X)_{E}:(F, E)\right.$ is a countable soft set $\}$, called soft ideal of countable soft subsets of $\tilde{X}$,

(5) $\tilde{I}_{(F, E)}=\left\{(G, E) \in S S(X)_{E}:(G, E) \tilde{\subseteq}(F, E)\right\}$.

(6) $\tilde{I}_{n}=\left\{(G, E) \in S S(X)_{E}: \operatorname{int}(\operatorname{cl}(G, E))=\tilde{\phi}\right\}$, called soft ideal of nowhere dense soft sets in $(X, \tau, E)$.

Theorem 3.1. Let $\tilde{I}$ be a soft ideal over a universe $X$. Then The collection $\tilde{I}_{e}=\{F(e):(F, E) \in \tilde{I}\}$ defines an ideal on $X$ for each $e \in E$. 
Proof. Let $F(e), G(e) \in \tilde{I}_{e}$. Then $\exists(F, E),(G, E) \in \tilde{I}$. Since $(F, E) \tilde{\cup}(G, E) \in \tilde{I}$ from Definition 3. Then $F(e) \cup G(e) \in \tilde{I}_{e}$. Now, let $F(e) \in \tilde{I}_{e}$ and $G(e) \subseteq X$ such that $G(e) \subseteq F(e)$. Then $\exists(F, E),(G, E) \in \tilde{I}$ such that $(G, E) \tilde{\tilde{C}}(F, E)$. It follows that $(G, E) \in \tilde{I}$. Thus $G(e) \in \tilde{I}_{e}$. This means that $\tilde{I}_{e}=\{F(e):(F, E) \in \tilde{I}\}$ defines an ideal on $X$ for each $e \in E$.

Corollary 3.1. A soft ideal over a universe $X$ with the same set of parameters $E$ is a parameterized collection of crisp ideals on $X$.

Proof. Obvious from Theorem 3.

Remark 3.1. The converse of theorem 3 is not true in general, as shown in the following example.

Example 3.2. Let $X=\left\{h_{1}, h_{2}, h_{3}\right\}, E=\left\{e_{1}, e_{2}\right\}$ and $\tilde{I}=\left\{\tilde{\phi},\left(F_{1}, E\right),\left(F_{2}, E\right),\left(F_{3}, E\right),\left(F_{4}, E\right)\right\} \quad$ where $\left(F_{1}, E\right),\left(F_{2}, E\right),\left(F_{3}, E\right),\left(F_{4}, E\right)$ are soft sets over $X$ defined as follows:

$F_{1}\left(e_{1}\right)=\left\{h_{2}\right\}, F_{1}\left(e_{2}\right)=\left\{h_{1}\right\}$,

$F_{2}\left(e_{1}\right)=\left\{h_{1}, h_{2}\right\}, F_{2}\left(e_{2}\right)=\left\{h_{3}\right\}$,

$F_{3}\left(e_{1}\right)=\left\{h_{2}\right\}, F_{3}\left(e_{2}\right)=\left\{h_{1}, h_{3}\right\}$,

$F_{4}\left(e_{1}\right)=\left\{h_{1}\right\}, \quad F_{4}\left(e_{2}\right)=\left\{h_{3}\right\} . \quad$ Then $\tilde{I}_{e_{1}}=\left\{\phi,\left\{h_{1}\right\},\left\{h_{2}\right\},\left\{h_{1}, h_{2}\right\}\right\} \quad$ and $\tilde{I}_{e_{2}}=\left\{\phi,\left\{h_{1}\right\},\left\{h_{3}\right\},\left\{h_{1}, h_{3}\right\}\right\}$ are ideals on $X$. However, $\tilde{I}$ is not soft ideal on $X$ because $\left(F_{1}, E\right) \tilde{\cup}\left(F_{2}, E\right)=(G, E)$, where $G\left(e_{1}\right)=\left\{h_{1}, h_{2}\right\}, G\left(e_{2}\right)=\left\{h_{1}, h_{3}\right\}$ and $(G, E) \notin \tilde{I}$.

Definition 3.2. Let $(X, \tau, E)$ be a soft topological space and $I$ be a soft ideal over $X$ with the same set of parameters $E$. Then

$(F, E)^{*}(\tilde{I}, \tau)\left(\operatorname{orF}_{E}^{*}\right)=\tilde{\cup}\left\{x_{e} \in \varepsilon: O_{x_{e}} \tilde{\cap}(F, E) \tilde{\notin} \tilde{I} \forall O_{x_{e}} \in \tau\right\}$ is called the soft local function of $(F, E)$ with respect to $\tilde{I}$ and $\tau$, where $O_{x_{e}}$ is a $\tau$-open soft set containing $x_{e}$.

Theorem 3.2. Let $\tilde{I}$ and $\tilde{J}$ be any two soft ideals with the same set of parameters $E$ on a soft topological space $(X, \tau, E)$. Let $(F, E),(G, E) \in S S(X)_{E}$. Then

(1) $(\tilde{\phi})^{*}=\tilde{\phi}$,

(2) $(F, E) \tilde{\subseteq}(G, E) \Rightarrow(F, E)^{*} \subseteq(G, E)^{*}$,

(3) $\tilde{I} \subseteq \tilde{J} \Rightarrow(F, E)^{*}(\tilde{J}) \subseteq(F, E)^{*}(\tilde{I})$,

(4) $(F, E)^{*} \tilde{\subseteq} c l(F, E)$, where $c l$ is the soft closure w.r.t. $\tau$,

(5) $(F, E)^{*}$ is $\tau$-closed soft set.

(6) $\left((F, E)^{*}\right)^{*} \tilde{\subseteq}(F, E)^{*}$,

(7) $((F, E) \tilde{\cup}(G, E))^{*}=(F, E)^{*} \cup(G, E)^{*}$,

(8) $\tilde{\cup}_{j}(F, E)^{*}=\left(\tilde{\cup}_{j}(F, E)\right)^{*}$,

(9) $((F, E) \tilde{\cap}(G, E))^{*} \subseteq(F, E)^{*} \cap(G, E)^{*}$,

$(10)(F, E)^{*}--\quad(G, E)^{*}$

$((F, E)-(G, E))^{*}-(G, E)^{*} \tilde{\subseteq}((F, E)-(G, E))^{*}$,

(11) $(G, E) \in \tau \Rightarrow(G, E) \tilde{\cap}(F, E)^{*}=$ $(G, E) \tilde{\cap}((G, E) \cap(F, E))^{*} \tilde{\subseteq}((G, E) \tilde{\cap}(F, E))^{*}$,
$(12)(H, E) \in \tilde{I} \Rightarrow((F, E) \tilde{\cup}(H, E))^{*}=(F, E)^{*}=((F, E)-$ $(H, E))^{*}$.

Proof.

(1)Obvious from Definition 3.

(2)Let $x_{e} \tilde{\in}(F, E)^{*}$. Then $O_{x_{e}} \tilde{\cap}(F, E) \notin \tilde{I} \forall O_{x_{e}} \in \tau$. Since $O_{x_{e}} \tilde{\cap}(F, E) \tilde{\subseteq} O_{x_{e}} \tilde{\cap}(G, E)$ and $O_{x_{e}} \tilde{\cap}(F, E) \notin \tilde{I}$. Then $O_{x_{e}} \tilde{\cap}(G, E) \notin \tilde{I} \forall O_{x_{e}} \in \tau$ from Definition 3.1. Hence $x_{e} \tilde{\in}(G, E)^{*}$. Thus $(F, E)^{*} \tilde{\subseteq}(G, E)^{*}$.

(3)Let $x_{e} \tilde{\in}(F, E)^{*}(\tilde{J})$. Then $O_{x_{e}} \tilde{\cap}(F, E) \notin \tilde{J} \forall O_{x_{e}} \in \tau$. Since $\tilde{I} \subseteq \tilde{J}$. Then $O_{x_{e}} \tilde{\cap}(F, E) \notin \tilde{I} \forall O_{x_{e}} \in \tau$. Hence $x_{e} \tilde{\in}(F, E)^{*}(\tilde{I})$. Thus $(F, E)^{*}(\tilde{J}) \tilde{\subseteq}(F, E)^{*}(\tilde{I})$.

(4)Assume that $x_{e} \tilde{\notin} c l(F, E)$. Then $\exists O_{x_{e}} \in \tau$ such that $O_{x_{e}} \tilde{\cap}(F, E)=\tilde{\phi} \in \tilde{I}$. Hence $x_{e} \tilde{\notin}(F, E)^{*}$. Thus $(F, E)^{*} \underline{\subseteq} c l(F, E)$.

(5)Clearly $(F, E)^{*} \tilde{\subseteq} c l(F, E)^{*}$. So let $x_{e} \tilde{\in} c l\left((F, E)^{*}\right)$. Then $\left.O_{x_{e}} \tilde{\cap}(F, E)^{*}\right) \neq \tilde{\phi} \forall O_{x_{e}} \in \tau$ from Theorem 2.1. Hence $\exists y_{e^{\prime}} \tilde{\in} O_{x_{e}} \tilde{\cap}(F, E)^{*}$. Thus $y_{e^{\prime}} \in O_{x_{e}}$ and $y_{e^{\prime}} \in(E, E)^{*}$. It follows that $O_{y_{e}^{\prime}} \tilde{\cap}(F, E) \notin \tilde{I} \forall O_{y_{e}^{\prime}} \in \tau$. This implies that $O_{x_{e}} \tilde{\cap}(F, E) \notin \tilde{I} \forall O_{x_{e}} \in \tau$. So $x_{e} \tilde{\in}(F, E)^{*}$. This means that $(F, E)^{*}=\operatorname{cl}(F, E)^{*}$ and consequently $\operatorname{cl}(F, E)^{*}=$ $(F, E)^{*}$.

(6)Since $\quad\left((F, E)^{*}\right)^{*} \tilde{\subseteq} c l(F, E)^{*} \quad$ from (4). Then $\left((F, E)^{*}\right)^{*} \tilde{\subseteq} c l(F, E)^{*}=(F, E)^{*}$ from $(5)$.

(7)Let $\quad x_{e} \tilde{\in}((F, E) \tilde{\cup}(G, E))^{*}$. Then $O_{x_{e}} \tilde{\cap}((F, E) \tilde{\cup}(G, E))$

$\left(O_{x_{e}} \tilde{\cap}(F, E)\right) \tilde{\cup}\left(O_{x_{e}} \tilde{\cap}(G, E)\right) \notin \tilde{I} \forall O_{x_{e}} \in \tau$. Hence either $O_{x_{e}} \tilde{\cap}(F, E) \notin \tilde{I}$ or $O_{x_{e}} \tilde{\cap}(G, E) \notin \tilde{I} \forall O_{x_{e}} \in \tau$ from Definition 3.1. This means that either $x_{e} \tilde{\in}(F, E)^{*}$ or $x_{e} \tilde{\in}(G, E)^{*}$. Thus $x_{e} \tilde{\in}(F, E)^{*} \cup(G, E)^{*}$. It follows that $((F, E) \tilde{\cup}(G, E))^{*} \tilde{\subseteq}(F, E)^{*} \cup(G, E)^{*}$. For the reverse inclusion, since $(F, E),(G, E) \tilde{\subseteq}((F, E) \tilde{\cup}(G, E)) . \quad$ Then $(F, E)^{*} \tilde{\subseteq}((F, E) \tilde{\cup}(G, E))^{*} \quad$ and $(G, E)^{*} \tilde{\subseteq}((F, E) \tilde{\cup}(G, E))^{*} \quad$ from (2). Hence $(F, E)^{*} \cup(G, E)^{*} \tilde{\subseteq}((F, E) \cup(G, E))^{*}$ and it is implies that $((F, E) \tilde{\cup}(G, E))^{*}=(F, E)^{*} \cup(G, E)^{*}$.

(8)Obvious from (7).

(9)Since $\quad((F, E) \tilde{\cap}(G, E)) \tilde{\subseteq}(F, E),(G, E)$. Then $((F, E) \tilde{\cap}(G, E))^{*} \tilde{\subseteq}(F, E)^{*}$ and $((F, E) \tilde{\cap}(G, E))$ $\tilde{\subseteq}(G, E)^{*} \quad$ from $\quad$ (2). Hence $((F, E) \tilde{\cap}(G, E))^{*} \tilde{\subseteq}(F, E)^{*} \tilde{\cap}(G, E)^{*}$.

(10)We first prove that $(F, E)^{*}-(G, E)^{*}=((F, E)-(G, E))^{*}-(G, E)^{*}$. Since $\quad(F, E)-(G, E) \tilde{\subseteq}(F, E)$. Then $((F, E) \quad-\quad(G, E))^{*} \tilde{\subseteq}(F, E)^{*}$. Hence $((F, E)-(G, E))^{*}-(G, E)^{*} \tilde{\subseteq}(F, E)^{*}-(G, E)^{*}$. For the reverse inclusion, since $(F, E)=[(F, E)-(G, E)] \tilde{\cup}[(F, E) \tilde{\cap}(G, E)] . \quad$ Then $(F, E)^{*}=[[(F, E)-(G, E)] \tilde{\cup}[(F, E) \tilde{\cap}(G, E)]]^{*}$. It follows 
$(F, E)^{*}=[[(F, E)-(G, E)] \tilde{\cup}[(F, E) \tilde{\cap}(G, E)]]^{*}=$ $[(F, E)-(G, E)]^{*} \tilde{\cup}[(F, E) \tilde{\cap}(G, E)]^{*} \tilde{\subseteq}[(F, E)-$ $(G, E)]^{*} \tilde{\cup}(G, E)^{*} \quad$ from (7). Hence $(F, E)^{*}-(G, E)^{*} \tilde{\subseteq}\left[[(F, E)-(G, E)]^{*} \tilde{\cup}(G, E)^{*}\right]-$ $(G, E)^{*}=\left[[(F, E)-(G, E)]^{*} \tilde{\cup}(G, E)^{*}\right] \tilde{\cap}(G, E)^{*^{\prime}}=$ $[(F, E) \quad-\quad(G, E)]^{*} \quad-\quad(G, E)^{*} . \quad$ Thus $(F, E)^{*}-(G, E)^{*}=((F, E)-(G, E))^{*}-(G, E)^{*}$. Also, if $x_{e} \tilde{E}((F, E)-(G, E))^{*}-(G, E)^{*}$. Then $x_{e} \tilde{\in}((F, E)-(G, E))^{*} \quad$ and $\quad$ consequently $(F, E)^{*} \quad-\quad(G, E)^{*}=$ $((F, E)-(G, E))^{*}-(G, E)^{*} \tilde{\subseteq}((F, E)-(G, E))^{*}$.

(11)Let $x_{e} \tilde{\epsilon}(G, E) \tilde{\cap}(F, E)^{*}$. Then $x_{e} \tilde{\in}(G, E)$ and $x_{e} \tilde{\in}(F, E)^{*}$. Hence $\left(O_{x_{e}} \tilde{\cap}(F, E)\right) \notin \tilde{I} \forall O_{x_{e}} \in \tau$. Since $(G, E)$ is $\tau$ - open soft set containing $x_{e}$. Then $(F, E) \tilde{\cap}(G, E)) \notin \tilde{I}$. Hence $\left(O_{x_{e}} \tilde{\cap}(G, E)\right) \tilde{\cap}(F, E) \notin \tilde{I}$, as $O_{x_{e}} \tilde{\cap}(G, E) \in \tau$. It follows that $O_{x_{e}} \tilde{\cap}((G, E) \tilde{\cap}(F, E)) \notin \tilde{I} \quad \forall O_{x_{e}} \in \tau$. Thus $x_{e} \tilde{\in}((G, E) \tilde{\cap}(F, E))^{*} \tilde{\cap}(F, E)^{*} \quad$ This implies that $(G, E) \tilde{\cap}(F, E)^{*} \tilde{\subseteq}(G, E) \tilde{\cap}$

$((G, E) \cap(F, E))^{*}$. For the reverse inclusion, since $((F, E) \tilde{\cap}(G, E)) \tilde{\subseteq}(F, E)$.

Then

$((F, E) \tilde{\cap}(G, E))^{*} \tilde{\subseteq}(F, E)^{*} \quad$ from $\quad$ (2). Hence $\left.(G, E) \tilde{\cap}((F, E) \tilde{\cap}(G, E))^{*} \tilde{\subseteq} G, E\right) \tilde{\cap}(F, E)^{*} . \quad$ Thus $(G, E) \tilde{\cap}(F, E)^{*}=(G, E) \tilde{\cap}((G, E) \cap(F, E))^{*}$. Also, if $x_{e} \tilde{\in}((G, E) \tilde{\cap}(F, E))^{*} \tilde{\cap}(F, E)$.

Then $x_{e} \tilde{\in}((G, E) \tilde{\cap}(F, E))^{*}$.

(12)We first prove that $((F, E) \tilde{\cup}(H, E))^{*}=(F, E)^{*}$. So let $x_{e} \tilde{\in}((H, E) \tilde{\cup}(F, E))^{*} . \quad$ Then $O_{x_{e}} \tilde{\cap}((H, E) \tilde{\cup}(F, E)) \quad \notin \quad \tilde{I} \forall \quad O_{x_{e}} \in \tau$. Hence $\left(O_{x_{e}} \tilde{\cap}(H, E)\right) \tilde{\cup}\left(O_{x_{e}} \tilde{\cap}(F, E)\right) \notin \tilde{I}$. It follows that either $O_{x_{e}} \tilde{\cap}(H, E) \notin \tilde{I} \quad$ or $\quad O_{x_{e}} \tilde{\cap}(F, E) \notin \tilde{I}$. But $O_{x_{e}} \tilde{\cap}(H, E) \notin \tilde{I}$ gives $(H, E) \in \tilde{I}$ which is a contradiction. Thus $O_{x_{e}} \tilde{\cap}(F, E) \notin \tilde{I} \forall O_{x_{e}} \in \tau$. So $x_{e} \tilde{\in}(F, E)^{*}$. For the reverse inclusion, since $(F, E) \tilde{\subseteq}((H, E) \tilde{\cup}(F, E))$.

Then

$(F, E)^{*} \tilde{\subseteq}((H, E) \tilde{\cup}(F, E))^{*}$ from (2).

Now we prove that $(F, E)^{*}=((F, E)-(H, E))^{*}$. Since $\quad((F, E)-(H, E)) \tilde{\subseteq}(F, E)$. Then $((F, E)-(H, E))^{*} \tilde{\subseteq}(F, E)^{*}$ from (2). For the reverse inclusion, let $x_{e} \tilde{\notin}((F, E)-(H, E))^{*}$. Then $\exists O_{x_{e}} \in \tau$ such that $O_{x_{e}} \tilde{\cap}((F, E)-(H, E)) \in \tilde{I}$. Since $(H, E) \in \tilde{I}$. Then $(H, E) \tilde{\cup}\left(O_{x_{e}} \tilde{\cap}((F, E)-(H, E))\right) \in \tilde{I}$. Hence $(H, E) \tilde{\cup}\left(O_{x_{e}} \tilde{\cap}(F, E)\right) \in \tilde{I}$. Thus $\left.O_{x_{e}} \tilde{\cap}(F, E)\right) \in \tilde{I}$ for some $O_{x_{e}} \in \tau$. It follows that $x_{e} \tilde{\notin}(F, E)^{*}$. This means that $(F, E)^{*} \tilde{\subseteq}((F, E)-(H, E))^{*}$. This completes the proof.
Theorem 3.3. Let $(X, \tau, E)$ be a soft topological space and $\tilde{I}$ be a soft ideal over $X$ with the same set of parameters $E$. Then the operator $c l^{*}: S S(X)_{E} \rightarrow S S(X)_{E}$ defined by:

$$
c l^{*}(F, E)=(F, E) \tilde{\cup}(F, E)^{*} .
$$

is a soft closure operator.

Proof. $c l^{*}(\tilde{\phi})=\tilde{\phi} \tilde{\cup}(\tilde{\phi})^{*}=\tilde{\phi} \tilde{\cup} \tilde{\phi}=\tilde{\phi}$ from Theorem $3.2(1)$, and obviously $(F, E) \tilde{\subseteq} c l^{*}(F, E)$

$\forall(F, E) \in S S(X)_{E}$. Now $c l^{*}((F, E) \tilde{\cup}(G, E))=$ $((F, E) \tilde{\cup}(G, E)) \tilde{\cup}((F, E) \tilde{\cup}(G, E))^{*}=((F, E) \tilde{\cup}$

$(G, E)) \cup\left((F, E)^{*} \cup(G, E)^{*}\right)$

$\left((F, E) \tilde{\cup}(F, E)^{*}\right) \tilde{\cup}\left((G, E) \widetilde{\cup}(G, E)^{*}\right)$

$c l^{*}(F, E) \tilde{\cup} c l^{*}(G, E)$ from Theorem $3.2(7)$. Also, for any $(F, E) \in S S(X)_{E}, c l^{*}\left(c l^{*}(F, E)\right)=c l^{*}\left((F, E) \cup(F, E)^{*}\right)=$ $\left((F, E) \tilde{\cup}(F, E)^{*}\right) \tilde{\cup}\left((F, E) \tilde{\cup}(F, E)^{*}\right)^{*}$

$\left((F, E) \tilde{\cup}(F, E)^{*}\right) \tilde{\cup}\left((F, E)^{*}\right.$

$\left.\left.\left((F, E)^{*}\right)^{*}\right) \tilde{\subseteq}\left((F, E) \tilde{\cup}(F, E)^{*}\right)\right) \tilde{\cup}$

$\left((F, E)^{*} \cup\left((F, E)^{*}\right)\right)=c l^{*}(F, E)$ from Theorem $3.2(6)$.

Definition 3.3. Let $(X, \tau, E)$ be a soft topological space, $\tilde{I}$ be a soft ideal over $X$ with the same set of parameters $E$ and $c l^{*}: S S(X)_{E} \rightarrow S S(X)_{E}$ be the soft closure operator. Then there exists a unique soft topology over $X$ with the same set of parameters $E$, finer than $\tau$, called the $*$-soft topology, denoted by $\tau^{*}(\tilde{I})$ or $\tau^{*}$, given by

$$
\tau^{*}(\tilde{I})=\left\{(F, E) \in S S(X)_{E}: c l^{*}(F, E)^{\prime}=(F, E)^{\prime}\right\} .
$$

\section{Example 3.2.}

(1)If $\quad \tilde{I}=\{\tilde{\phi}\}, \quad$ then $(F, E)^{*}(\tilde{I}, \tau)=\operatorname{cl}(F, E) \forall(F, E) \in S S(X)_{E}$. Hence $C l^{*}(F, E)=\operatorname{cl}(F, E)$ and $\tau^{*}=\tau$.

(2)If $\tilde{I}=S S(X)_{E}$, then $(F, E)^{*}(\tilde{I}, \tau)=\tilde{\phi} \forall(F, E) \in S S(X)_{E}$. Hence $C l^{*}(F, E)=(F, E)$ and $\tau^{*}=S S(X)_{E}$ (the soft discrete topology).

(3)If $\tilde{I} \tilde{\subseteq} \tilde{J}$, then $(F, E)^{*}(\tilde{J}, \tau) \tilde{\subseteq}(F, E)^{*}(\tilde{I}, \tau)$. Hence the $\star$ soft topological space $\left(X, \tau^{*}(\tilde{J}), E\right)$ is finer than the $\star$ soft topological space $\left(X, \tau^{*}(\tilde{I}), E\right)$.

(4)Let $X=\left\{h_{1}, h_{2}, h_{3}\right\}, E=\{e\}$ and $\tau=\{\tilde{X}, \tilde{\phi},(F, E)\}$ where $(F, E)$ is a soft set over $X$ defined by $F(e)=$ $\left\{h_{2}\right\}$. Then $\tau$ defines a soft topology on $X$. Let $\tilde{I}=$ $\{\tilde{\phi},(G, E)\}$ be a soft ideal over $X$ where $(G, E)$ is a soft set over $X$ defined by $G(e)=\left\{h_{2}\right\}$. Then $\tau^{*}=$ $\left\{\tilde{X}, \tilde{\phi},\left(F_{1}, E\right),\left(F_{2}, E\right)\right\}$ where $\left(F_{1}, E\right),\left(F_{2}, E\right)$ are soft sets over $X$ where $F_{1}(e)=\left\{h_{2}\right\}$ and $F_{2}(e)=\left\{h_{1}, h_{3}\right\}$.

(5)Let $\quad X=\left\{h_{1}, h_{2}, h_{3}\right\}, \quad E=\{e\} \quad$ and $\tau=\left\{\tilde{X}, \tilde{\phi},\left(F_{1}, E\right),\left(F_{2}, E\right)\right\}$ where $\left(F_{1}, E\right),\left(F_{2}, E\right)$ are soft sets over $X$ defined defined as follows:

$F_{1}(e)=\left\{h_{2}\right\}, F_{2}(e)=\left\{h_{1}, h_{2}\right\}$. Then $\tau$ defines a soft topology on $X$. Let $\tilde{I}=\left\{\tilde{\phi},\left(G_{1}, E\right),\left(G_{2}, E\right),\left(G_{3}, E\right)\right\}$ be a soft ideal over $X$ where $\left(G_{1}, E\right),\left(G_{2}, E\right),\left(G_{3}, E\right)$ 
are soft sets over $X$ defined by $G_{1}(e)=\left\{h_{1}\right\}$, $G_{2}(e)=\left\{h_{2}\right\}$ and $G_{3}(e)=\left\{h_{1}, h_{2}\right\}$. Then $\tau^{*}=S S(X)_{E}$.

(6)Let $X=\left\{h_{1}, h_{2}\right\}, \quad E=\left\{e_{1}, e_{2}\right\} \quad$ and $\tau=\left\{\tilde{X}, \tilde{\phi},\left(F_{1}, E\right),\left(F_{2}, E\right)\right\}$ where $\left(F_{1}, E\right),\left(F_{2}, E\right)$ are soft sets over $X$ defined defined as follows:

$F_{1}\left(e_{1}\right)=\left\{h_{1}\right\}, F_{1}\left(e_{2}\right)=\left\{h_{2}\right\}$,

$F_{2}\left(e_{1}\right)=X, F_{2}\left(e_{2}\right)=\left\{h_{2}\right\}$. Then $\tau$ defines a soft topology on $X$. Let $\tilde{I}=\left\{\tilde{\phi},\left(G_{1}, E\right),\left(G_{2}, E\right)\right.$,

$\left.\left(G_{3}, E\right)\right\}$ be a soft ideal over $X$ where $\left(G_{1}, E\right),\left(G_{2}, E\right),\left(G_{3}, E\right)$ are soft sets over $X$ defined by $G_{1}\left(e_{1}\right)=\phi, G_{1}\left(e_{2}\right)=\left\{h_{1}\right\}$,

$G_{2}\left(e_{1}\right)=\left\{h_{1}\right\}, G_{2}\left(e_{2}\right)=\phi$,

and $G_{3}\left(e_{1}\right)=\left\{h_{1}\right\}, G_{3}\left(e_{2}\right)=\left\{h_{1}\right\}$. Then $\tau^{*}=\left\{\tilde{X}, \tilde{\phi},\left(F_{1}, E\right),\left(F_{2}, E\right),\left(F_{3}, E\right),\left(F_{4}, E\right),\left(F_{5}, E\right)\right.$,

$\left(F_{6}, E\right),\left(F_{7}, E\right),\left(F_{8}, E\right),\left(F_{9}, E\right)$,

$\left.\left(F_{10}, E\right),\left(F_{11}, E\right),\left(F_{12}, E\right)\right\}$ is a soft topology finer than $\tau$, where $\left(F_{1}, E\right),\left(F_{2}, E\right),\left(F_{3}, E\right),\left(F_{4}, E\right)$,

$\left(F_{5}, E\right),\left(F_{6}, E\right),\left(F_{7}, E\right),\left(F_{8}, E\right),\left(F_{9}, E\right)$,

$\left(F_{10}, E\right),\left(F_{11}, E\right)$ are soft sets over $X$ defined defined as follows:

$F_{1}\left(e_{1}\right)=\left\{h_{2}\right\}, F_{1}\left(e_{2}\right)=X$,

$F_{2}\left(e_{1}\right)=\left\{h_{2}\right\}, F_{2}\left(e_{2}\right)=\left\{h_{2}\right\}$,

$F_{3}\left(e_{1}\right)=\left\{h_{1}\right\}, F_{3}\left(e_{2}\right)=X$,

$F_{4}\left(e_{1}\right)=\left\{h_{1}\right\}, F_{4}\left(e_{2}\right)=\left\{h_{2}\right\}$,

$F_{5}\left(e_{1}\right)=X, F_{5}\left(e_{2}\right)=\left\{h_{2}\right\}$

$F_{6}\left(e_{1}\right)=X, F_{6}\left(e_{2}\right)=\phi$,

$F_{7}\left(e_{1}\right)=\phi, F_{7}\left(e_{2}\right)=X$,

$F_{8}\left(e_{1}\right)=\left\{h_{1}\right\}, F_{8}\left(e_{2}\right)=\phi$,

$F_{9}\left(e_{1}\right)=\phi, F_{9}\left(e_{2}\right)=\left\{h_{2}\right\}$,

$F_{10}\left(e_{1}\right)=\phi, F_{10}\left(e_{2}\right)=\phi$

$F_{11}\left(e_{1}\right)=X, F_{11}\left(e_{2}\right)=X$.

Theorem 3.4. Let $(X, \tau, E)$ be a soft topological space and $\tilde{I}$ be a soft ideal over $X$ with the same set of parameters $E$. Then

$$
\beta(\tilde{I}, \tau)=\{(F, E)-(G, E):(F, E) \in \tau,(G, E) \in \tilde{I}\}
$$

is a soft basis for the soft topology $\tau^{*}(\tilde{I})$.

Proof. Since $\tilde{X} \in \tau, \tilde{\phi} \in \tilde{I}$. Then $\tilde{X}-\tilde{\phi} \in \beta$. Hence $\tilde{X} \in \beta$ and $\tilde{U}_{j \in J}\left(\left(F_{j}, E\right)-\left(G_{j}, E\right)\right)=\tilde{X}$. Also, let $\left(\left(F_{1}, E\right)-\left(G_{1}, E\right)\right),\left(\left(F_{2}, E\right)-\left(G_{2}, E\right)\right) \in \beta$ such that $x_{e} \tilde{\in}\left(\left(F_{1}, E\right)-\left(G_{1}, E\right)\right) \tilde{\cap}\left(\left(F_{2}, E\right)-\left(G_{2}, E\right)\right)$. Then $x_{e} \tilde{\in}\left(\left(F_{1}, E\right)-\left(G_{1}, E\right)\right) \tilde{\cap}\left(\left(F_{2}, E\right)-\left(G_{2}, E\right)\right)=$ $\left(\left(F_{1}, E\right) \tilde{\cap}\left(F_{2}, E\right)\right)-\left(\left(G_{1}, E\right) \tilde{\cup}\left(G_{2}, E\right)\right) \in \beta(\tilde{I}, \tau)$. Thus $\beta$ is a soft basis of $\tau^{*}$.

Corollary 3.2. Let $(X, \tau, E)$ be a soft topological space and $\tilde{I}$ be a soft ideal over $X$ with the same set of parameters $E$. Then $\tau \subseteq \beta(\tilde{I}, \tau) \subseteq \tau^{*}(\tilde{I})$.

Proof. Immediate from Theorem 3.2 (3) and Theorem 3.4. Theorem 3.5. Let $(X, \tau, E)$ be a soft topological space and $\tilde{I}$ be a soft ideal over $X$ with the same set of parameters $E$. If $(F, E)^{d},(F, E)^{d^{*}}$ are the derived soft sets of $(F, E)$ in $(X, \tau, E)$ and $\left(X, \tau^{*}(\tilde{I}), E\right)$ respectively, then $(F, E)^{d^{*}} \tilde{\subseteq}(F, E)^{d}$.

\section{Proof.}

Let $x_{e} \tilde{\in}(F, E)^{d^{*}}$ if and only if $x_{e} \tilde{\epsilon} c l^{*}\left((F, E)-x_{e}\right)$ if and only if $x_{e} \tilde{\in}\left[\left((F, E)-x_{e}\right)\right] \tilde{\cup}\left[\left((F, E)-x_{e}\right)\right]^{*}$ if and only if $\left(O_{x_{e}}-x_{e}\right) \tilde{\cap}(F, E) \notin \tilde{I} \forall O_{x_{e}} \in \tau$. Then $x_{e} \tilde{\in}(F, E)^{d}$.

\section{Compatibility of soft ideals with soft topology}

Definition 4.1. Let $(X, \tau, E)$ be a soft topological space and $\tilde{I}$ be a soft ideal over $X$ with the same set of parameters $E$. We say that the soft topology $\tau$ is compatible with the soft ideal $\tilde{I}$, denoted by $\tau \sim \tilde{I}$, if the following holds for every $(F, E) \in S S(X)_{E}$ :

if for every soft point $x_{e}, x_{e} \in(F, E)$ there exists $O_{x_{e}}$ such that $O_{x_{e}} \tilde{\cap}(F, E) \in \tilde{I}$, then $(F, E) \in \tilde{I}$.

Theorem 4.1. Let $(X, \tau, E)$ be a soft topological space, $\tilde{I}$ be a soft ideal over $X$ with the same set of parameters $E$ and $\tau \sim \tilde{I}$. Then the following are equivalent:

(1)For every $(F, E) \in S S(X)_{E},(F, E) \tilde{\cap}(F, E)^{*}=\tilde{\phi}$, then $(F, E)=\tilde{\phi}$.

(2)For every $(F, E) \in S S(X)_{E},\left((F, E)-(F, E)^{*}\right)^{*}=\tilde{\phi}$.

(3)For every $(F, E) \in S S(X)_{E}$, $\left((F, E) \tilde{\cap}(F, E)^{*}\right)^{*}=(F, E)^{*}$.

Proof.

$(1) \Longrightarrow(2)$ Let $\quad(F, E) \in S S(X)_{E} . \quad$ Since $\left((F, E)-(F, E)^{*}\right) \tilde{\cap}\left((F, E)-(F, E)^{*}\right)^{*}=\tilde{\phi}$. Then $\left((F, E)-(F, E)^{*}\right)^{*}=\tilde{\phi}$ by $(1)$.

$(2) \Longrightarrow(3)$ Let $(F, E) \in S S(X)_{E}$. Since $(F, E)=$ $\left((F, E)-\left((F, E) \tilde{\cap}(F, E)^{*}\right)\right) \tilde{\cup}\left((F, E) \tilde{\cap}(F, E)^{*}\right)$. Then $(F, E)^{*}=$ $\left[(F, E)-\left((F, E) \tilde{\cap}(F, E)^{*}\right) \tilde{\cup}\left((F, E) \tilde{\cap}(F, E)^{*}\right)\right]^{*}=$ $\left[(F, E)-\left((F, E) \tilde{\cap}(F, E)^{*}\right)\right]^{*}$

$\tilde{\cup}\left[(F, E) \tilde{\cap}(F, E)^{*}\right]^{*}=\tilde{\phi} \tilde{\cup}\left[(F, E) \tilde{\cap}(F, E)^{*}\right]^{*}=$ $\left[(F, E) \tilde{\cap}(F, E)^{*}\right]^{*}$ by $(2)$.

(3) $\Longrightarrow(1)$ Let $(F, E) \in S S(X)_{E}$ and $(F, E) \tilde{\cap}(F, E)^{*}=\tilde{\phi}$. Then $(F, E)^{*}=\left[(F, E) \tilde{\cap}(F, E)^{*}\right]^{*}=(\tilde{\phi})^{*}=\tilde{\phi}$. Hence $(F, E) \in \tilde{I}$.

Corollary 4.1. Let $(X, \tau, E)$ be a soft topological space, $\tilde{I}$ be a soft ideal over $X$ with the same set of parameters $E$, $(F, E) \in S S(X)_{E}$ and $\tau \sim \tilde{I}$. Then $\left((F, E)^{*}\right)^{*}=(F, E)^{*}$.

Proof. Let $(F, E) \in S S(X)_{E}$. Since $\left.(F, E)^{*}=\left((F, E) \tilde{\cap}(F, E)^{*}\right)^{*} \tilde{\subseteq}(F, E)^{*}\right)^{*}$ from Theorem 4.1 
(3). But we have $\left((F, E)^{*}\right)^{*} \tilde{\subseteq}(F, E)^{*}$ from Theorem 3.2 (6). Thus $\left((F, E)^{*}\right)^{*}=(F, E)^{*}$.

Theorem 4.2. Let $(X, \tau, E)$ be a soft topological space and $\tilde{I}$ be a soft ideal over $X$ with the same set of parameters $E$. Then the following are equivalent:

(1) $\tau \sim \tilde{I}$

(2)For every $(F, E) \in S S(X)_{E}$ such that $(F, E) \tilde{\cap}(F, E)^{*}=$ $\tilde{\phi},(F, E) \in \tilde{I}$.

(3)For every $(F, E) \in S S(X)_{E},(F, E)-(F, E)^{*} \in \tilde{I}$.

(4)For every $\tau^{*}$-closed soft subset $(F, E)$, $(F, E)-(F, E)^{*} \in \tilde{I}$.

(5)For every $(F, E) \in S S(X)_{E}$, if $(F, E)$ contains no nonnull soft set $(G, E)$ with $(G, E) \tilde{\subseteq}(G, E)^{*}$, then $(F, E) \in$ $\tilde{I}$.

\section{Proof.}

$(1) \Longrightarrow(2)$ Let $\quad(F, E) \in S S(X)_{E} \quad$ such that $(F, E) \tilde{\cap}(F, E)^{*}=\tilde{\phi}$. Then $\forall \quad x_{e} \tilde{\in}(F, E)$ and $x_{e} \tilde{\notin}(F, E)^{*}$, we have $O_{x_{e}} \tilde{\cap}(F, E) \in \tilde{I}$ for some $O_{x_{e}} \in \tau$. Thus $(F, E) \in \tilde{I}$ by (1).

$(2) \Longrightarrow(3)$ Let $\quad(F, E) \in S S(X)_{E}$. Since $\left((F, E)-(F, E)^{*}\right) \tilde{\cap}\left((F, E)-(F, E)^{*}\right)^{*}=$ $\left((F, E) \tilde{\cap}(F, E)^{*^{\prime}}\right) \tilde{\cap}$

$\left((F, E)-(F, E)^{*}\right)^{*} \tilde{\subseteq}\left((F, E) \tilde{\cap}(F, E)^{*^{\prime}}\right) \tilde{\cap}(F, E)^{*}=\tilde{\phi}$. Then $(F, E)-(F, E)^{*} \in \tilde{I}$ by (2).

$(3) \Longrightarrow(4)$ Let $(F, E)$ be a $\tau^{*}$-closed soft subset. Then $(F, E) \in S S(X)_{E}$. Hence $(F, E)-(F, E)^{*} \in \tilde{I}$ by (3).

$(4) \Longrightarrow(1)$ Let $(F, E) \in S S(X)_{E}$ and assume that for every $x_{e} \tilde{\in}(F, E)$ there exists $O_{x_{e}}$ such that $O_{x_{e}} \tilde{\cap}(F, E) \in \tilde{I}$. Then $x_{e} \tilde{\notin}(F, E)^{*}$. Hence $\left((F, E) \tilde{\cap}(F, E)^{*}\right)=\tilde{\phi}$ and since $(F, E) \tilde{\cup}(F, E)^{*}$ is $\tau^{*}$-closed soft set, we have $\left((F, E) \tilde{\cup}(F, E)^{*}\right)-\left((F, E) \tilde{\cup}(F, E)^{*}\right)^{*} \in \tilde{I}$ by $(4)$. Hence $\left((F, E) \tilde{\cup}(F, E)^{*}\right)-\left((F, E)^{*} \tilde{\cup}\left((F, E)^{*}\right)^{*}\right)=$ $\left((F, E) \sim(F, E)^{*}\right)-\left((F, E)^{*}\right)=(F, E) \in \tilde{I}$ by Theorem $3.2(6,7)$. Thus $\tau \sim \tilde{I}$.

$(3) \Longrightarrow(5)$ Let $(F, E) \in S S(X)_{E}$ such that $(F, E)$ contains no non-null soft set $(G, E)$ with $(G, E) \tilde{\subseteq}(G, E)^{*}$. Since $(F, E) \tilde{\cap}(F, E)^{*} \tilde{\subseteq}(F, E)^{*}=\left((F, E) \tilde{\cap}(F, E)^{*}\right)^{*}$ from Theorem 4.1 (3). It follows that $(F, E) \tilde{\cap}(F, E)^{*} \tilde{\simeq}\left((F, E) \tilde{\cap}(F, E)^{*}\right)^{*}$. By assumption, $(F, E) \tilde{\cap}(F, E)^{*}=\tilde{\phi}$. Thus $(F, E)=(F, E)-(F, E)^{*} \in \tilde{I}$ by $(3)$.

$(5) \Longrightarrow(3)$ Let $\quad(F, E) \in S S(X)_{E} . \quad$ Since $\left((F, E)-(F, E)^{*}\right) \tilde{\cap}\left((F, E)-(F, E)^{*}\right)^{*}=\tilde{\phi} \quad$ and $\left.(F, E)-(F, E)^{*}\right)$ contains no non-null soft set $(G, E)$ with $(G, E) \tilde{\subseteq}(G, E)^{*}$. Hence $(F, E)-(F, E)^{*} \in \tilde{I}$ by (5).

Theorem 4.3. If $(X, \tau, E)$ is a soft topological space, $\tilde{I}$ be a soft ideal over $X$ with the same set of parameters $E$ and compatible with $\tau$. Then a soft set is $\tau^{*}$-closed if and only if it is the union of a $\tau$-closed soft set and a soft set in $\tilde{I}$.

Proof.

Let $(F, E)$ be a $\tau^{*}$-closed soft set. Then $c l^{*}(F, E)=(F, E)$ and $(F, E) \tilde{\cup}(F, E)^{*}=(F, E)$. Hence $(F, E)^{*} \tilde{\subseteq}(F, E)$. Thus $(F, E)=\left((F, E)-(F, E)^{*}\right) \tilde{\cup}(F, E)^{*}$, $(F, E)-(F, E)^{*} \in \tilde{I}$ from Theorem 4.2 and $(F, E)^{*}$ is $\tau$-closed soft set from Theorem 3.2 (5). Conversely, let $(F, E)=(G, E) \tilde{\cup}(I, E)$, where $(G, E)$ is $\tau$-closed soft set and $(I, E) \in \tilde{I}$. Then $(F, E)^{*}=((G, E)-(I, E))^{*}=$ $(G, E)^{*} \tilde{\simeq} c l(G, E)=(G, E) \underline{\simeq}(F, E)$ from Theorem 3.2 $(4,12)$. Hence $(F, E) \tilde{\cup}(F, E)^{*}=(F, E)$. Thus $c l^{*}(F, E)=(F, E)$. It follows that $(F, E)$ is a $\tau^{*}$-closed soft set.

Theorem 4.4. Let $(X, \tau, E)$ be a soft topological space, $\tilde{I}$ be a soft ideal over $X$ with the same set of parameters $E$. If $\tau \sim \tilde{I}$, then $\beta(\tilde{I}, \tau)$ is a soft topology and hence $\beta=\tau^{*}$.

Proof. Let $(G, E) \in \tau^{*}$. Then $(G, E)^{\prime}=(F, E) \tilde{\cup}(I, E)$, where $(F, E)$ is $\tau$-closed soft set and $(I, E) \in \tilde{I}$. Hence $(G, E)=(X, E)-((F, E) \tilde{\cup}(I, E))=((X, E)-$ $(F, E)) \tilde{\cap}((X, E)-(I, E))=((X, E)-(F, E))-(I, E)$, where $(F, E) \in \tau$ and $(I, E) \in \tilde{I}$. Thus $(G, E)=((X, E)-(F, E))-(I, E) \in \beta(\tilde{I}, \tau)$. This means that $\tau^{*} \subseteq \beta(\tilde{I}, \tau)$. But $\beta(\tilde{I}, \tau) \subseteq \tau^{*}(\tilde{I})$ from Corollary 3.2. It follows that $\beta=\tau^{*}$.

\section{Acknowledgements}

The authors express their sincere thanks to the reviewers for their careful checking of the details and for helpful comments that improved this paper. The authors are also thankful to the editors-in-chief and managing editors for their important comments which helped to improve the presentation of the paper.

\section{References}

[1] B. Ahmad and A. Kharal, On fuzzy soft sets, Advances in Fuzzy Systems, 1-6 (2009).

[2] H. Aktas and N. agman, Soft sets and soft groups, Information Sciences, 1, 2726-2735 (2007).

[3] M. I. Ali, F. Feng, X. Liu, W. K. Min and M. Shabir, On some new operations in soft set theory, Computers and Mathematics with Applications, 57, 1547-1553 (2009).

[4] A. Aygnoglu and H. Aygn, Introduction to fuzzy soft groups, Computers and Mathematics with Applications, 58, 12791286 (2009).

[5] Banu Pazar Varol and Halis Aygun, On soft Hausdorff spaces, Annals of Fuzzy Mathematics and Informatics, 5, 15-24 (2013). 
[6] N. agman, F.itak and S. Enginoglu, Fuzzy parameterized fuzzy soft set theory and its applications, Turkish Journal of Fuzzy Systems, 1, 21-35 (2010).

[7] N.agman and S. Enginoglu, Soft set theory and uniint decision making, European Journal of Operational Research, 207, 848-855 (2010).

[8] N.agman, S. Enginoglu and S. Karatas, Soft topology, Computers and Mathematics with Applications, 62, 351-358 (2011).

[9] S. Hussain and B. Ahmad, Some properties of soft topological spaces, Comput. Math. Appl., 62, 4058-4067 (2011).

[10] D. Jankovic and T.R. Hamlet, New topologies from old via ideals, The American Mathematical Monthly, 97, 295-310 (1990).

[11] A.Kandil, O. A. E. Tantawy, S. A. El-Sheikh and A. M. Abd El-latif, $\gamma$-operation and decompositions of some forms of soft continuity in soft topological spaces. To appear in the journal Annals of Fuzzy Mathematics and Informatics (AFMI).

[12] K. Kannan, Soft generalized closed sets in soft topological spaces, Journal of Theoretical and Applied Technology, 37, 17-21 (2012).

[13] D. V. Kovkov, V. M. Kolbanov and D. A. Molodtsov, Soft sets theory-based optimization, Journal of Computer and Systems Sciences International, 46, 872-880 (2007).

[14] P. K. Maji, R. Biswas and A. R. Roy, Fuzzy soft sets, Journal of Fuzzy Mathematics, 9, 589-602 (2001).

[15] P. K. Maji, R. Biswas and A. R. Roy, Intuitionistic fuzzy soft sets, Journal of Fuzzy Mathematics, 9, 677-691 (2001).

[16] P. K. Maji,R. Biswas and A. R. Roy, Soft set theory, Computers and Mathematics with Applications, 45, 555-562 (2003).

[17] P. Majumdar and S. K. Samanta, Generalised fuzzy soft sets, Computers and Mathematics with Applications, 59, 14251432 (2010).

[18] D.Molodtsov, V. Y. Leonov and D. V. Kovkov, Soft sets technique and its application, Nechetkie Sistemy i Myagkie Vychisleniya, 1, 8-39 (2006).

[19] D. A. Molodtsov, Soft set theory-firs tresults, Computers and Mathematics with Applications, 37, 19-31 (1999).

[20] A. Mukherjee and S. B. Chakraborty, On intuitionistic fuzzy soft relations, Bulletin of Kerala Mathematics Association, 5, 35-42 (2008).

[21] Sk. Nazmul and S. K. Samanta, Neighbourhood properties of soft topological spaces, Annals of Fuzzy Mathematics and Informatics, 6, 1-15 (2012).

[22] D. Pei and D. Miao, From soft sets to information systems, in: X. Hu, Q. Liu, A. Skowron, T. Y. Lin, R. R. Yager, B. Zhang (Eds.), Proceedings of Granular Computing, in: IEEE, 2, 617-621 (2005).

[23] M. Shabir and M. Naz, On soft topological spaces, Comput. Math. Appl., 61, 1786-1799 (2011).
[24] Sujoy Das and S. K. Samanta, Soft metric, Annals of Fuzzy Mathematics and Informatics, 6, 77-94 (2013).

[25] Weijian Rong, The countabilities of soft topological spaces, International Journal of Computational and Mathematical Sciences, 6, 159-162 (2012).

[26] W. Xu, J. Ma, S. Wang and G. Hao, Vague soft sets and their properties, Computers and Mathematics with Applications, 59, 787-794 (2010).

[27] Y. Zou and Z. Xiao, Data analysis approaches of soft sets under incomplete information, Knowledge-Based Systems, 21, 941-945 (2008).

[28] I. Zorlutuna, M. Akdag, W.K. Min and S. Atmaca, Remarks on soft topological spaces, Annals of Fuzzy Mathematics and Informatics, 3, 171-185 (2012).

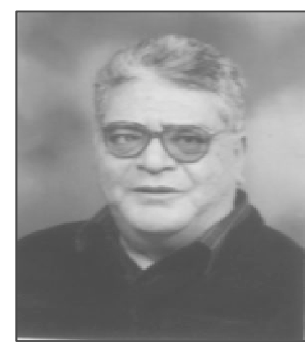

Ali Kandil Saad Ibrahim is a Professor of Mathematics at Helwan University. $\mathrm{He}$ received the Ph.D. degree in Topology from the University of Moscow in 1978. His primary research areas are General Topology, Fuzzy Topology, double sets and theory of sets. Dr. Kandil has published over 80 papers in refereed journals and contributed several book chapters in various types of Mathematics textbooks. He is a Fellow of the Egyptian Mathematical Society and Egyptian Physics Mathematical Society. He was the Supervisor of 20 PHD and about 30 MSC students.

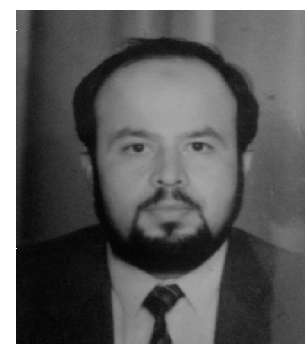

Osama Abd El-Hamid El-Tantawy is a Professor of Mathematics at Zagazig University. He was born in 1951. He received the Ph.D. degree in Topology from the University of Zagazig in 1988. His primary research areas are General Topology, Fuzzy Topology, double sets and theory of sets. Dr. Osama has published over 50 papers in refereed journals. He is a Fellow of the Egyptian Mathematical Society and Egyptian Physics Mathematical Society. He was the Supervisor of 10 PHD and about 17 MSC students. 


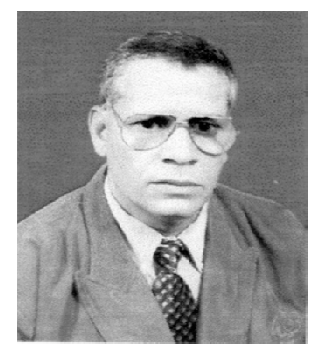

Sobhy
Aly El-Sheikh is an
assistance Professor of
pure Mathematics, Ain
Shams University, Faculty
of Education, Mathematic
Department, Cairo, Egypt.
He was born in 1955.

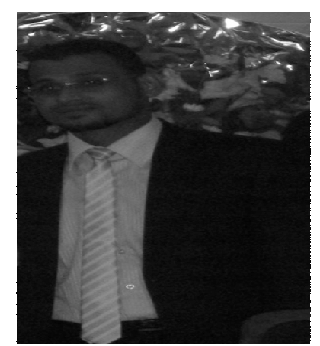

Alaa

Mohamed

Abd El-Latif Daby is a Ph.D student in pure Mathematics (Topology) in Ain Shams University, Faculty of Education, Mathematic Department, Cairo, Egypt. $\mathrm{He}$ was born in 1985 . He He received the $\mathrm{Ph} . \mathrm{D}$. degree in Topology from the University of Zagazig. His primary research areas are General Topology, Fuzzy Topology, double sets and theory of sets. Dr. Sobhy has published over 15 papers in Fuzzy set and system Journal (FSS), Information science Journal (INFS), Journal of fuzzy Mathematics and Egyptian Journal of Mathematical Society. He was the Supervisor of many PHD MSC Thesis. received the MSC Thesis degree in Topology from Ain Shams University in 2012. His primary research areas are General Topology, Bitopology and Ditopology. 